\title{
CAPÍTULO 07: FERMENTAÇÃO EM ESTADO SÓLIDO E SUBMERSO PARA PRODUÇÃO DE CELULASE: REVISÃO BIBLIOGRÁFICA
}

\author{
CAPÍTULO 07: FERMENTACIÓN SÓLIDA Y SUMERGIDA PARA LA \\ PRODUCCIÓN DE CELULASAS: REVISIÓN BIBLIOGRÁFICA
}

\section{CHAPTER 07: SOLID AND SUBMERGED FERMENTATION FOR CELLULASE PRODUCTION: BIBLIOGRAPHIC REVIEW}

\author{
Letícia Souza Silva $^{1}$; Adryelen Cassiano Martins ${ }^{2}$; Larine Kupski³; Juliana Bueno Ruiz ${ }^{4}$
}

DOI: https://doi.org/10.31692/978-65-88970-19-5.98-112

\begin{abstract}
RESUMO
Os processos fermentativos em estado sólido (FES) e submerso (FS) consistem na degradação de glicose ou de nutrientes por meio de microrganismos, como fungos, bactérias e leveduras. Estes tipos de fermentação são amplamente aplicados para a produção de enzimas, como a celulase. A alta eficiência do processo fermentativo para a produção de celulase está diretamente ligada as condições adequadas de cultivo. O presente trabalho teve como objetivo realizar uma revisão para identificar as condições de fermentação utilizadas na Fermentação em Estado Sólido (FES) e Fermentação Submersa (FS) para a produção da celulase. Foram selecionados um total de 21 artigos, todos entre os anos de 2018 a 2020, onde foram analisados fatores: microrganismos, substratos, $\mathrm{pH}$, umidade, temperatura e agitação. As seguintes ferramentas de dados bibliográficas foram utilizadas: Scielo, Science Direct, Scopus e Web of Science. Além disso as referências foram gerenciadas pelo software de banco de dados "EndNote" para a exclusão por duplicidade. A pesquisa dos artigos foi realizada com base nas palavras-chave: cellulase OR cellulolytic, Solid-State fermentation, Submerged fermentation e Production OR Synthesis. Os microrganismos que mais se destacaram foram Aspergillus nigger e Trichoderma reesei para as FES e FS, respectivamente. Os substratos mais empregados foram os resíduos agroindustriais, evidenciandose o farelo do trigo. As condições de umidade, temperatura, $\mathrm{pH}$ e agitação tiveram diferenças tanto para FES quanto para FS, isso justifica pela diversidade de microrsganismo utilizados. Os dados demonstraram que existe uma grande quantidade de condições a serem trabalhadas e que muitas vezes as mesmas condições podem ser utilizadas para os dois tipos de fermentação para a produção de celulase. Palavras-Chave: Enzima; Fermentação; Substrato; Microrganismos.
\end{abstract}

\section{RESUMEN}

Los procesos de fermentación en estado sólido (FES) y sumergido (FS) consisten en la degradación de glucosa o nutrientes por medio de microorganismos, como hongos, bacterias y levaduras. Estos tipos de fermentación se aplican ampliamente para la producción de enzimas, como la celulasa. La alta eficiencia del proceso de fermentación para la producción de celulasa está directamente relacionada con las condiciones adecuadas de cultivo. El presente trabajo tuvo como objetivo realizar una revisión para identificar las condiciones de fermentación utilizadas en la fermentación en estado sólido (FES) y la fermentación sumergida (FS) para la producción de celulasa. Se seleccionaron un total de 21 artículos, todos entre los años 2018 a 2020, donde se analizaron factores: microorganismos, sustratos, pH, humedad, temperatura y agitación. Se utilizaron las siguientes herramientas de datos bibliográficos: Scielo, Science Direct, Scopus y Web of Science. Además, las referencias fueron gestionadas por el software de base de datos "EndNote" para su exclusión por duplicación. La búsqueda de los artículos se realizó en base a las palabras clave: cellulase OR cellulolytic, Solid-State fermentation, Submerged fermentation y Production OR Synthesis. Los microorganismos que más destacaron fueron Aspergillus nigger y Trichoderma reesei para la FES y FS, respectivamente. Los sustratos más utilizados fueron los residuos agroindustriales, evidenciando el salvado de trigo. Las condiciones de humedad, temperatura,

\footnotetext{
${ }^{1}$ Graduanda em Engenharia de Alimentos, Universidade Estadual de Maringá, lesouzasilva1995@gmail.com

${ }^{2}$ Graduanda em Engenharia de Alimentos, Universidade Estadual de Maringá,, adryelencassiano15@gmail.com

${ }^{3}$ Doutora em Engenharia e Ciências de Alimentos, Universidade Estadual de Maringá, lkupski@uem.br

${ }^{4}$ Doutora em Genética e Melhoramento, Universidade Estadual de Maringá, jbrrebecca2@uem.br
} 
pH y agitación presentaron diferencias tanto para FES como para FS, esto se justifica por la diversidad de microorganismos utilizados. Los datos demostraron que hay un gran número de condiciones en las que trabajar y que a menudo se pueden utilizar las mismas condiciones para ambos tipos de fermentación para la producción de celulasa.

Palabras Clave: Enzima; Fermentación; Sustrato; Microorganismos.

\begin{abstract}
The fermentation processes in solid state (FES) and submerged (FS) consist of the degradation of glucose or nutrients by means of microorganisms, such as fungi, bacteria and yeasts. These types of fermentation are widely applied for the production of enzymes, such as cellulase. The high efficiency of the fermentation process for the production of cellulase is directly linked to the appropriate conditions of cultivation. The present work aimed to carry out a review to identify the fermentation conditions used in Solid State Fermentation (FES) and Submerged Fermentation (FS) for the production of cellulase. A total of 21 articles were selected, all between the years 2018 to 2020, where factors were analyzed: microorganisms, substrates, $\mathrm{pH}$, humidity, temperature and agitation. The following bibliographic data tools were used: Scielo, Science Direct, Scopus and Web of Science. In addition, the references were managed by the database software "EndNote" for exclusion due to duplication. The search for the articles was carried out based on the keywords: cellulase OR cellulolytic, Solid-State fermentation, Submerged fermentation and Production OR Synthesis. The microorganisms that stood out the most were Aspergillus nigger and Trichoderma reesei for the FES and FS, respectively. The most used substrates were the agro-industrial residues, evidencing the wheat bran. The conditions of humidity, temperature, $\mathrm{pH}$ and agitation had differences for both FES and FS, this justifies by the diversity of microorganisms used. The data demonstrated that there are a great number of conditions to be worked on and that the same conditions can often be used for both types of fermentation for the production of cellulase.
\end{abstract}

Keywords: Enzyme; Fermentation; Substrate; Microorganisms.

\title{
INTRODUÇÃO
}

As celulases são empregadas em inúmeros processos nas indústrias, como extração de óleos vegetais, extração de suco de frutas, agente de coloração alimentar, redução de deterioração de alimentos, pré-tratamento de chá fermentado, extração de café e açúcar, biocombustível, indústria têxtil, biogás, fabricação de papel, fármacos, tratamento de resíduos, entre outros (BAHRAMIAN et al., 2010; BEHERA et al., 2016; BEHERA et al., 2017; HU et al., 2018; SILVA, et al., 2019).

A produção de celulases microbianas é realizada a partir de processos fermentativos, onde destacam-se a Fermentação Submersa (FS) e Fermentação em Estado Sólido (FES) (CUNHA et al., 2014). O custo de produção de enzimas microbianas é fortemente ligado à sua produtividade (SHELDO e PELT, 2013; FLORENCIO et al., 2017). O custo dos substratos e as variações de rendimento enzimático, são problemas economicamente determinantes para produção de celulase, prejudicando seu potencial para aplicação em escala industrial (LIMAYEM e RICKE, 2012). Assim, para que o processo se torne viável é necessário que os parâmetros de otimização do processo como a seleção do microrganismo ideal para a enzima de interesse, o meio de cultivo, $\mathrm{pH}$, temperatura, aeração e agitação sejam definidos para se 
O tipo de microrganismos estudado, bem como o substrato para essa produção tornamse importante, pois a enzima celulase é indutiva, portanto, a sua produtividade enzimática está diretamente ligada a quantidade de celulose presente nos substratos e quais os microrganismos conseguem produzir a máxima atividade a partir dos mesmos (SUTO e TOMITA, 2001).

Portanto, pelas vantagens e desvantagens da produção de celulase pelas fermentações sólida e submersa, o objetivo desta revisão é avaliar as condições ótimas (substrato, microrganismo, umidade, temperatura, $\mathrm{pH}$, agitação) utilizadas em ambos processos.

\section{FUNDAMENTAÇÃO TEÓRICA}

As enzimas são substâncias orgânicas de polímeros de aminoácidos que atuam como catalizadores biológicos, ou seja, acelerando velocidade das reações (SOARES et al., 2010). A produção de enzimas é uma área amplamente promissora, estima-se que em 2023 o mercado global movimentará cerca de US\$ 7 bilhões, uma taxa de crescimento anual de 4,9\% no período de 2018-2023 (ARUN, 2017). Isto em virtudes das suas novas tecnologias para agregar valor em síntese de compostos (ROVEDA et al., 2010), além de serem consideradas ambientalmente corretas pela substituição de catalisadores químicos, economia de energia pela utilização de temperaturas, pH e pressão brandas (THOMAS et al., 2013; YAZID et al., 2017).

As enzimas podem ser obtidas de fontes vegetais, animais e microbianas. As enzimas microbianas podem ser produzidas através de processos fermentativos (submerso e estado sólido) (CUNHA et al., 2014) e apresentam vantagens frente as vegetais e animais, como baixos custos de produção, viabilidade de larga escala em fermentadores industriais, distintas características físico-químicas, são diretamente ligadas ao hábitat e fisiologia do microrganismo produtor, altamente sensíveis a modificações genética e são um recurso renovável (KASANA e GULATI, 2011). Dentre as enzimas microbianas produzidas destacam-se as celulases (ABREU et al., 2015).

As celulases constituem um complexo enzimático formado por endo- $\beta$-1,4-glicanases (EC 3.2.1.4), exo- $\beta$-1,4-glicanases (EC 3.2.1.91) e $\beta$-1,4-glicanases (EC 3.2.1.21) que atuam sinergisticamente na conversão da celulose cristalina a glicose (GOMES et al., 2016). A atividade enzimática de cada componente do complexo enzimático pode ser determinada suas atividades separadamente, ou a ação sinérgica pode ser determinada, empregando o ensaio de hidrólise no papel filtro e expressando a atividade de FPase (RAWAT et al., 2014).

A produção dessas enzimas é realizada a partir de processos fermentativos (CUNHA 
et al., 2014). A FS é a mais utilizada, devido ao fácil controle de processos e possibilita a sua

utilização em escalas industriais (LIU et al., 2020), para este tipo de fermentação utiliza-se água, que permite minimizar os gradientes de temperatura, concentrar os nutrientes (SILVA et al, 2019), além de ser possível a realização de aeração, agitação e controle do pH (RAMAMOORTHY et al., 2019). A FES, no entanto, é um processo de crescimento microbiano em um substrato sólido com pouca ou nenhuma água livre aparente (SOCCOL et al., 2017), de biorreatores simples, baixo custo de matéria-prima e de produção, requisitos de energia reduzidos, mínima produção de águas residuais, alta produtividade e alta eficiência recuperação do produto (SADH et al., 2018). Para que isso seja possível existem uma gama de microrganismos capazes de degradar materiais celulósicos como fungos ou bactérias SILVA et al., 2019).

Segundo Kuhad et al. (2016), a produção de celulase por meio processos fermentativos envolve uma diversidade de microrganismos, dentre eles são amplamente utilizados fungos e bactérias (SALAZAR et al., 2019). Alguns microrganismos, como as bactérias, necessitam de um alto teor de umidade para seu crescimento e são mais indicadas a FS (IRFAN et al., 2019). Por outro lado, a fermentação por fungos embora seja amplamente recomendada aos dois tipos de processo, sólido e submerso (SUBSAMRAN et al., 2019), é mais adequado para FES, por oferecer menor teor de umidade e sustentar o crescimento de fungos em materiais lignocelulósicos (YOON et al., 2014).

\section{METODOLOGIA}

A pesquisa é de abordagem quali e quantitativa e quanto aos procedimentos técnicos utilizou-se de revisão bibliográfica. As seguintes ferramentas de dados bibliográficas foram utilizadas: Scielo, Science Direct, Scopus e Web of Science. Para todas as buscas foram utilizadas base de dados apenas para artigos de pesquisa publicados em 2018, 2019 e 2020. Além disso, as referências foram gerenciadas pelo software de banco de dados "EndNote" para a exclusão por duplicidade.

A pesquisa dos artigos foi realizada com base nas palavras-chave: cellulase $O R$ cellulolytic, Solid-State fermentation, Submerged fermentation e Production OR Synthesis. Os dados selecionados englobaram tipo de fermentação, condições ótimas de fermentação (substrato, microrganismo, umidade, temperatura, $\mathrm{pH}$, agitação). 
Todos os artigos incluídos na pesquisa tiveram suas publicações de 2018 a 2020 e avaliaram a produção de celulase (expressão com atividade de FPase) por meio de processos fermentativos com planejamentos experimentais de otimização. Dentre eles 13 [1, 6, 8, 10, 11, $12,13,14,15,17,19,20,21]$ foram conduzidos por meio de fermentação sólida e 8 [2, 3, 4, 5 , $7,9,16,18]$ por meio de fermentação submersa.

A maior quantidade de artigos encontrados para fermentação em estado solido (n=13), frente a submersa $(n=8)$ pode estar associado ao fato que na FS há melhor controle de fatores ambientais como temperatura, gradiente de nutrientes e $\mathrm{pH}$, sendo tradicionalmente utilizada para produzir enzimas em escalas industriais (SINGHANIA et al., 2010). Controlar esses parâmetros ainda é considerado um desafio da FES (ABDULLAH et al., 2018), embora tenha um bom rendimento enzimático, e forneça um ambiente de crescimento em condições adequadas para que os microrganismos se desenvolverem naturalmente (CUNHA et al., 2012), portanto, mais estudos são conduzidos com este modo de cultivo a fim de contribuir com novas estratégias para produção enzimática.

$\mathrm{Na}$ Tabela 01 estão descritas as informações sobre as características gerais abordadas pelos os artigos selecionados.

Tabela 01: Condições da fermentação em estado sólida (FES) e submersa (FS) dos estudos selecionados para revisão da produçao de celulase, entre os anos de 2018 a $2020(n=21)$

\begin{tabular}{|c|c|c|c|c|c|c|c|c|}
\hline \multirow{2}{*}{$\begin{array}{l}\text { Código } \\
\text { do } \\
\text { artigo }\end{array}$} & \multirow{2}{*}{ Processo } & \multicolumn{6}{|c|}{ Condições ótimas de fermentação } & \multirow[b]{2}{*}{ Referêncas } \\
\hline & & Substrato & Microrganismo & $\begin{array}{l}\text { Umidade } \\
(\%)\end{array}$ & $\begin{array}{c}\mathrm{T} \\
\left({ }^{\circ} \mathrm{C}\right)\end{array}$ & $\mathrm{pH}$ & $\begin{array}{l}\text { Agitação } \\
\text { (rpm) }\end{array}$ & \\
\hline 1 & FES & $\begin{array}{c}\text { Bagaço de cana- } \\
\text { de -açúcar }\end{array}$ & Aspergillus niger & 80 & 35 & 3,50 & - & $\begin{array}{l}\text { Abdullah et al. } \\
\text { (2018) }\end{array}$ \\
\hline 2 & FS & $\begin{array}{l}\text { Mesocarpo de } \\
\text { coco }\end{array}$ & Trichoderma reesei & 100 & 30 & 5,50 & 150 & Dey et al. (2018) \\
\hline 3 & FS & Farelo de trigo & Schizophyllum commune & 100 & 25 & 5,00 & 100 & $\begin{array}{l}\text { Kumar et al. } \\
\text { (2018) }\end{array}$ \\
\hline 4 & FS & $\begin{array}{c}\text { Resíduos de } \\
\text { papel de } \\
\text { escritório }\end{array}$ & Bacillus velezensis & 100 & 30 & 4,70 & 100 & Nair et al. (2018) \\
\hline 5 & FS & - & Aspergillus $s p$ & 100 & 30 & 5,50 & 100 & $\begin{array}{l}\text { Shawky et al. } \\
\text { (2018) }\end{array}$ \\
\hline 6 & FES & $\begin{array}{l}\text { Resíduo têxtil } \\
\text { cottón/poliéster }\end{array}$ & Aspergillus niger & 78 & 28 & 7,29 & - & $\begin{array}{l}\text { Hu et al. } \\
(2018)\end{array}$ \\
\hline 7 & FS & Palha de arroz & Thermoascus aurantiacus & 100 & 50 & 5,00 & 120 & $\begin{array}{l}\text { Singh e Bajar } \\
\text { (2019) }\end{array}$ \\
\hline 8 & FES & Casca de soja & Penicillium sp. & 70 & 50 & 4,90 & - & $\begin{array}{l}\text { Salazar et al. } \\
\text { (2019) }\end{array}$ \\
\hline
\end{tabular}




\begin{tabular}{|c|c|c|c|c|c|c|c|c|}
\hline 9 & FS & $\begin{array}{c}\text { Casaca de } \\
\text { ervilha }\end{array}$ & Trichoderma reesei & 100 & 30 & 5,00 & 110 & $\begin{array}{l}\text { Sirohi et al. } \\
\text { (2019) }\end{array}$ \\
\hline 10 & FES & $\begin{array}{c}\text { Folhas de } \\
\text { plantação de } \\
\text { óleo de palma }\end{array}$ & Aspergillus niger & 75 & 30 & 4,50 & - & Tai et al. (2019) \\
\hline 11 & FES & Grama Vetiver & Aspergillus tubingensis & 80 & 30 & 7,00 & - & $\begin{array}{c}\text { Subsamran et al. } \\
\text { (2019) }\end{array}$ \\
\hline 12 & FES & $\begin{array}{c}\text { Farelo de } \\
\text { arroz, casca de } \\
\text { arroz e palha } \\
\text { de arroz }\end{array}$ & Trichoderma reesei & 75 & 35 & - & - & $\begin{array}{l}\text { Darabzadeh et } \\
\text { al. (2019) }\end{array}$ \\
\hline 13 & FES & $\begin{array}{c}\text { Vagens de } \\
\text { sementes de } \\
\text { Bombyx ceiba }\end{array}$ & Trichoderma viride & - & 35 & - & - & $\begin{array}{l}\text { Nazir et al } \\
\quad(2019)\end{array}$ \\
\hline 14 & FES & $\begin{array}{l}\text { Bagaço de } \\
\text { sorgo doce e } \\
\text { farelo trigo }\end{array}$ & Aspergillus terreus & 80 & 45 & 5,80 & - & $\begin{array}{l}\text { Sharma et al } \\
\text { (2019) }\end{array}$ \\
\hline 15 & FES & Farelo de trigo & Aspergillus niger & 76 & 30 & 5,00 & - & $\begin{array}{c}\text { Verma e Kumar } \\
\text { (2019) }\end{array}$ \\
\hline 16 & FS & $\begin{array}{c}\text { Casca de } \\
\text { amendoim }\end{array}$ & Bacillus paralichniformis & 100 & 35 & - & - & $\begin{array}{c}\text { Irfan et al } \\
(2020)\end{array}$ \\
\hline 17 & FES & Palha de arroz & Myceliophthora thermophila & 80 & 45 & 5,00 & - & $\begin{array}{c}\text { Kumar et al } \\
\quad(2020)\end{array}$ \\
\hline 18 & FS & $\begin{array}{l}\text { Dreche } \\
\text { (residuos } \\
\text { cereais } \\
\text { cervejeira) }\end{array}$ & Trichoderma reesei & 100 & 30 & 5,00 & 180 & $\begin{array}{c}\text { Cekmecelioglu } \\
\text { e Demirci } \\
(2020)\end{array}$ \\
\hline \multirow{2}{*}{19} & \multirow{2}{*}{ FES } & \multirow{2}{*}{ Farelo de trigo } & Trichoderma reesei & 61 & 30 & 5,00 & \multirow{2}{*}{ - } & \multirow[t]{2}{*}{$\begin{array}{l}\text { Verma e Kumar } \\
\quad(2020)\end{array}$} \\
\hline & & & Neurospora crassa & 66 & 30 & 6,00 & & \\
\hline 20 & FES & Farelo de trigo & Pestalotiopsis microspora & 80 & 32 & 4,75 & - & $\begin{array}{c}\text { Joukanapalle et } \\
\text { al (2020) }\end{array}$ \\
\hline 21 & FES & $\begin{array}{c}\text { Casca de } \\
\text { laranja, bagaço } \\
\text { de cana e } \\
\text { farelo de trigo }\end{array}$ & Aspergillus fumigatus & 65 & 31 & 7,00 & - & $\begin{array}{l}\text { Mondal et al } \\
\quad(2020)\end{array}$ \\
\hline
\end{tabular}

Fonte: Própria (2020).

De acordo com a Tabela 01, as condições ótimas de fermentação para a umidade na fermentação em estado sólido ficaram entre 61-80\% dependendo das diferentes condições. Quanto a temperatura, observou-se que a maioria dos artigos estudados estabeleceram a temperatura entre $30-35^{\circ} \mathrm{C}(\mathrm{n}=9)$ para FS e para FES $(\mathrm{n}=7)$. Pode-se notar que a solução nutriente apresentou uma grande diversidade para ambos os tipos de fermentação, o que está relacionado com o tipo de substrato e microrganismo utilizado que também apresentaram uma grande variedade. Para o valor de $\mathrm{pH}$, somente três estudos não mencionaram o valor e a maioria $(\mathrm{n}=10)$ utilizaram valores próximos de $\mathrm{pH} 5,0$, não apresentando grandes diferenças entre a FS e FES.

Essas variáveis se destacam, pois, um dos grandes desafios dos processos fermentativos em estado sólido é o controle de fatores ambientais, como temperatura, $\mathrm{pH}$ e umidade durante a fermentação (DEY et al., 2018), e seleção do microrganismo adequado está diretamente ligada a eficiência na produtividade enzimática (SUBSAMRA et al. 2019). Na fermentação submersa por sua vez a concentração de solução nutriente e substratos também se torna importante, pois o fato de operar em meio submerso ocasiona a diluição dos componentes presentes, sendo 
necessária a garantia das quantidades adequadas para o metabolismo microbiano e desenvolvimento do produto (DEY eet al., 2018; IRFAN et al., 2019).

Além das variáveis descritas anteriormente, o tipo de microrganismos estudado, bem como o substrato para essa produção tornam-se importante, pois a enzima celulase é indutiva, portanto, a sua produtividade enzimática está diretamente ligada a quantidade de celulose presente nos substratos e quais os microrganismos conseguem produzir a máxima atividade a partir dos mesmos (SUTO e TOMITA, 2001). Portanto, o efeito dessas variáveis serão discutidas isoladamente, bem como as produtividades obtidas.

Na Figura 01 estão descritos os diferentes microrganismos utilizados nos processos fermentativos (estado sólido e submerso) para produção de celulase.

Figura 01: Microrganismos utilizados para produção de celulase nas fermentações em estado sólido e submerso nos artigos selecionados com período de publicação entre os anos de 2018 a 2020.

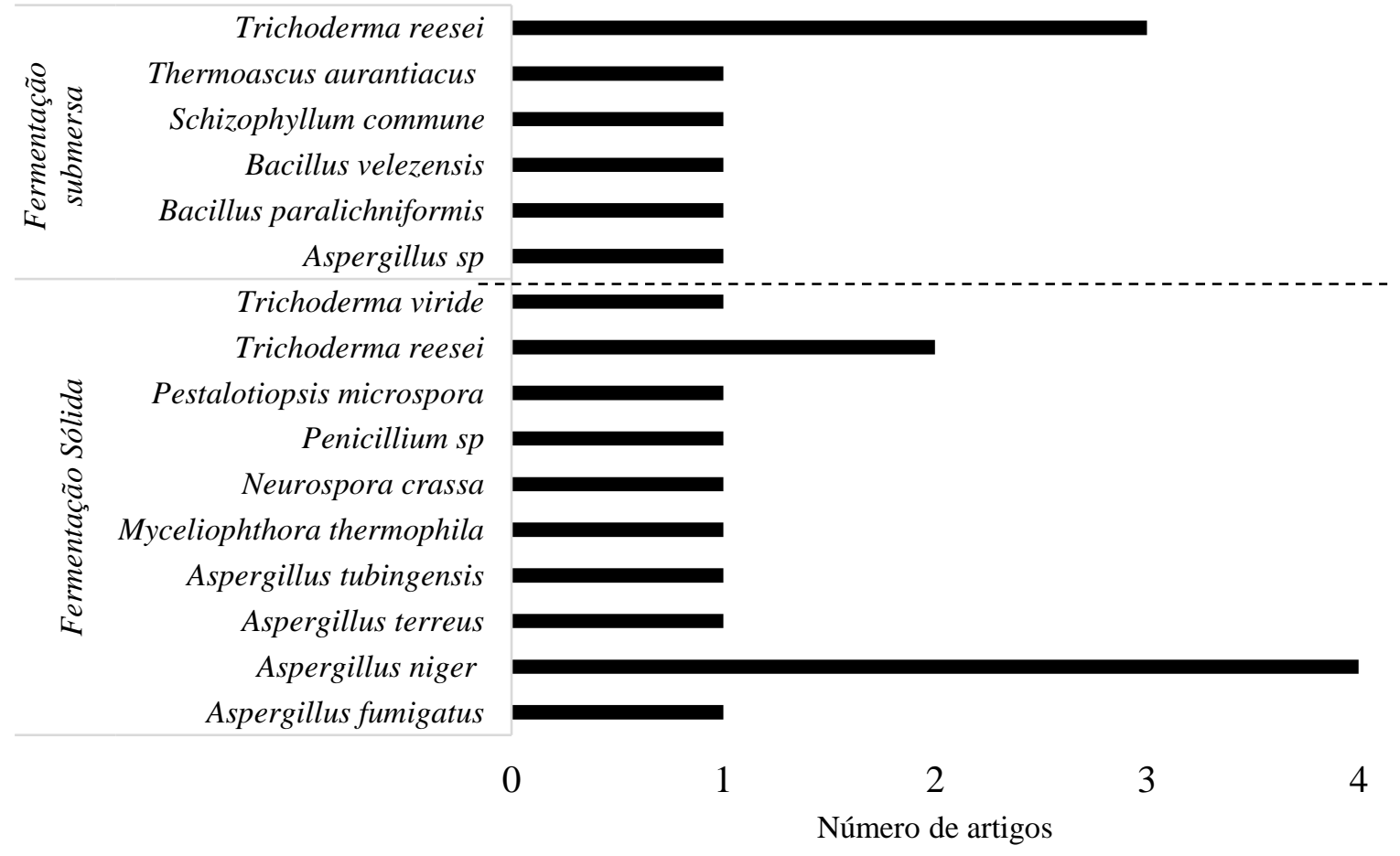

Fonte: Própria (2020).

No que se refere a utilização de microrganismos para processos fermentativos sólidos (FES) e submersos (FS), há uma notável variedade de espécies microbianas, como indicado na Figura 02.

Nos artigos selecionados na presente revisão ambos os processos apresentam maior número $(\mathrm{n}=19)$ de estudos utilizando fungos filamentosos, enquanto o uso de bactérias foi observado somente para a fermentação submersa $(n=2)$. Nos estudos de fermentação em estado 
sólido houve uma maior utilização do microrganismo A. niger $(\mathrm{n}=4)$, enquanto no processo de fermentação submersa o microrganismo mais utilizado foi o T. reesei $(\mathrm{n}=3)$

As cepas fúngicas A. niger e T. ressei tem-se mostrado importantes produtores de celulase, pela sua alta produtividade e aplicabilidade segura (ZNAMEROSKI et al. 2012). Alguns estudos apontam que fermentação submersa tem sido tradicionalmente bem-sucedida para a produção de celulase por meio de T. reesei em substratos lignocelulósicos (SATEESH et al., 2012; RANA et al., 2014; LI et al., 2016), assim como a fermentação em estado sólido com A. niger empregando substratos celulósicos (SANTOS et al., 2018), pelo seu rápido desenvolvimento e multiplicação farta de esporos (BLASZCZYK et al., 2014).

Os substratos selecionados pelos estudos são resíduos lignocelulósicos, como pode-se observar pela Figura 02:

Figura 02: Substratos utilizados para produção de celulase nas fermentações em estado sólido e submerso nos artigos selecionados com período de publicação entre os anos de 2018 a 2020.

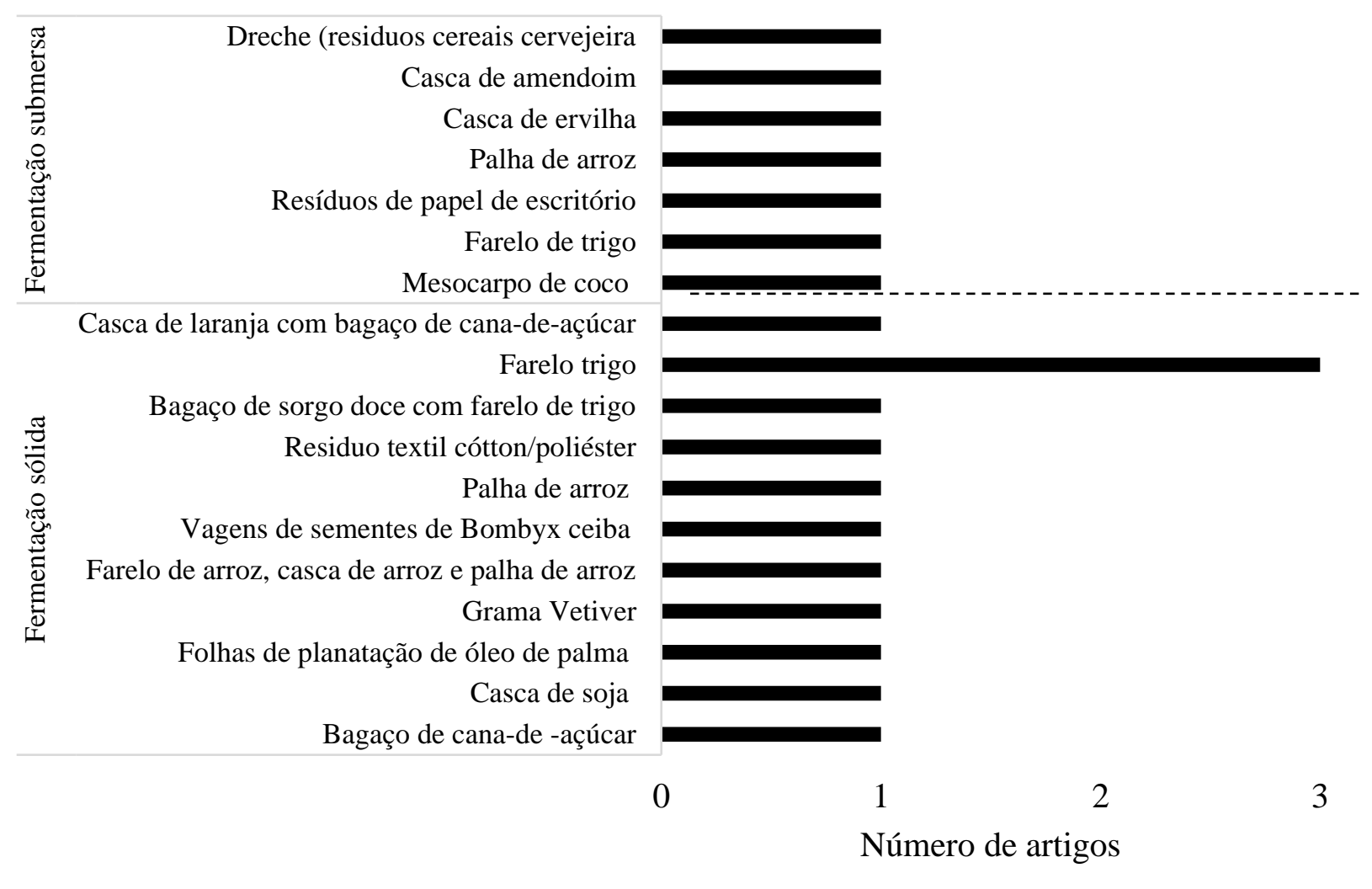

Fonte: Própria (2020).

Sabendo que a celulase é uma enzima indutiva, os substratos escolhidos para a fermentação são de extrema importância para a produção enzimática. Embora os resíduos sólidos sejam mais utilizados para a fermentação em estado sólido, nota-se que nos estudos 
selecionados para a revisão tanto para a FES quanto para a FS foram executadas a partir dos mesmos, como demonstrado na Figura 02.

Resíduos são uma alternativa promissora para redução de custos e aumento da sustentabilidade nos processos fermentativos para produção de enzimas celulase (SALAZAR et al., 2019). Estes resíduos são compostos pela chamada biomassa lignocelulósica, que apresentam, por sua vez, uma composição de aproximadamente 40-50\% de celulose, 25-30\% de hemicelulose e 15-20\% de lignina (ZHOU et al., 2017). Neste contexto, devido ao seu rico conteúdo orgânico, baixo custo e alta disponibilidade, os resíduos agrícolas podem ser substratos ideais para a fermentação microbiana (FALKOSKI et al., 2013; EL-BAKRY et al., 2015).

A FS (n=7) exibe, de acordo com a Figura 2, distintos tipos de resíduos, sendo eles resíduos agroindustriais $(n=6)$ e resíduos industrial $(n=1)$, resíduo de papel de escritório $(n=1)$. O artigo 5 de FS não foi incluído para esta análise, pois não apresentou o substrato usado em seu estudo. Para a FES (n=13), também é possível notar que uma maior parte dos substratos são resíduos agroindustriais $(n=12)$ e apenas um é resíduo industrial, resíduo têxtil de cótton/poliéster. Porém o resíduo de farelo de trigo é utilizado em maior quantidade, tanto individualmente $(n=3)$ quanto para misturas $(n=1)$, este mesmo acontecimento é verificado para o bagaço de cana-de-açúcar $(\mathrm{n}=2)$.

Ainda que a FS utilize resíduos agrícolas sólidos na sua composição do meio, para a disponibilidade de celulose (CEKMECELIOGLU e DEMIRCI, 2020), sua proporção será sempre reduzida, levando em consideração que este terá que ser combinado com um outro meio líquido (IRFAN et al., 2019). Já a FES os resíduos serão utilizados sem esta diluição, portanto a disponibilidade de celulose para a produção de celulase será maior (NAZIR et al., 2019).

Dentre os resíduos, o mais utilizado foi o farelo de trigo, que possui características em sua composição que o fazem um excelente indutor para produção de celulase, como os elevados níveis de proteínas (13-19\%) e hemicelulose (3-6\%), celulose (11\%) juntamente a um baixo teor de lignina (3-6\%) (FALKOSKI et al., 2013; CERDA et al., 2017; VERMA e KUMAR 2019). Ainda é possível notar que todos os 21 estudos foram combinados os substratos a uma solução nutriente que também auxiliarão na disposição de nutrientes para os microrganismos, sendo a mais utilizada pelos estudos a de Mendel $\left(\mathrm{KH}_{2} \mathrm{PO}_{4},\left(\mathrm{NH}_{4}\right)_{2} \mathrm{SO}_{4}, \mathrm{CO}\left(\mathrm{NH}_{2}\right)_{2}\right.$, $\mathrm{MgSO}_{4} .7 \mathrm{H}_{2} \mathrm{O}, \mathrm{CaCl}_{2}, \mathrm{FeSO}_{4} .7 \mathrm{H}_{2} \mathrm{O}, \mathrm{MnSO}_{4} \cdot \mathrm{H}_{2} \mathrm{O}, \mathrm{CoCl}_{2}, \mathrm{ZnSO}_{4} .7 \mathrm{H}_{2} \mathrm{O}$ ), alguns estudos a pontam alta utilização dessa solução nutriente pela sua alta eficiência na produção de celulase (MARTINS et al., 2007; SUN et al., 2018; CAMASSOLA et al., 2010). 
Diante dos artigos selecionados observou-se uma grande variação no tipo de microrganismo e substrato utilizado para a produção de celulase. Os microrganismos que mais se destacaram foram Aspergillus niger e Trichoderma reesei para as FES e FS, respectivamente. Os substratos mais empregados foram os resíduos agroindustriais, evidenciando-se o farelo do trigo. Quanto a temperatura, observou-se que a maioria dos artigos estudados estabeleceram a temperatura entre $30-35^{\circ} \mathrm{C}(\mathrm{n}=9)$ para FS e para FES $(\mathrm{n}=7)$. Os valores de $\mathrm{pH}$ utilizados nos estudos foram próximos de $\mathrm{pH}$ 5,0, não apresentando grandes diferenças entre a FS e FES.

Importante destacar que tanto para a FES quanto para a FS, o microrganismo Trichoderma reesei foi utilizado demonstrando que ele tem facilidade no crescimento em ambos os tipos de condução de fermentação. A utilização de substrato também apresentou uma grande variedade podendo estar relacionada com o tipo do microrganismo e as condições de fermentação utilizadas, o que demonstra uma grande diversidade de subprodutos agroindustriais que podem ser reutilizados para produção de celulase.

\section{REFERENCIAS}

ABDULLAH, Abdullah et al. Optimization of cellulase production by Aspergillus niger ITBCC L74 with bagasse as substrate using response surface methodology. HAYATI Journal of Biosciences, v. 25, n. 3, p. 115-115, 2018.

ABU YAZID, NORAZIAH et al. Solid-state fermentation as a novel paradigm for organic waste valorization: a review. Sustainability, v. 9, n. 2, p. 224, 2017.

ARUN, H. K. Global Markets for Enzymes in Industrial Applications (Report Highlights. BCC Research. Disponível em: < https://www.bccresearch.com/marketresearch/biotechnology/global-markets-for-enzymes-inindustrial-applications.html>.Acesso em: 28 outubro 2020. (2017) 1-184.

BAHRAMIAN, SAMIRA et al. Optimization of enzymatic extraction of sugars from Kabkab date fruit. Middle East J. Sci. Res., v. 7, p. 211-216, 2011.

BEHERA, SUDHANSHU S.; RAY, RAMESH C. Solid state fermentation for production of microbial cellulases: recent advances and improvement strategies. International journal of biological macromolecules, v. 86, p. 656-669, 2016.

BLASZCZYK, LMSKS et al. Trichoderma spp.-application and prospects for use in organic farming and industry. Journal of plant protection research, v. 54, n. 4, 2014.

CAMASSOLA, MARLI; DILLON, ALDO JP. Cellulases and xylanases production by Penicillium echinulatum grown on sugar cane bagasse in solid-state fermentation. Applied biochemistry and biotechnology, v. 162, n. 7, p. 1889-1900, 2010. 
CEKMECELIOGLU, DENIZ; DEMIRCI, ALI. Production of cellulase and xylanase enzymes using distillers dried grains with solubles (DDGS) by Trichoderma reesei at shake-flask scale and the validation in the benchtop scale bioreactor. Waste and Biomass Valorization, p. 1-10, 2020.

CERDA, ALEJANDRA et al. Cellulase and xylanase production at pilot scale by solid-state fermentation from coffee husk using specialized consortia: The consistency of the process and the microbial communities involved. Bioresource technology, v. 243, p. 1059-1068, 2017.

CUNHA, F. M. et al. Liquefaction of sugarcane bagasse for enzyme production. Bioresource technology, v. 172, p. 249-252, 2014.

CUNHA, F. M. et al. Sequential solid-state and submerged cultivation of Aspergillus niger on sugarcane bagasse for the production of cellulase. Bioresource technology, v. 112, p. 270-274, 2012.

DA SILVA, ISADORA FERREIRA et al. High-yield cellulase and LiP production after SSF of agricultural wastes by Pleurotus ostreatus using different surfactants. Biocatalysis and Agricultural Biotechnology, v. 22, p. 101428, 2019.

DARABZADEH, NAZANIN; HAMIDI-ESFAHANI, ZOHREH; HEJAZI, PARISA. Optimization of cellulase production under solid-state fermentation by a new mutant strain of Trichoderma reesei. Food science \& nutrition, v. 7, n. 2, p. 572-578, 2019.

DE ABREU, JÉSSICA ALINE SOARES; ROVIDA, AMANDA FLÁVIA DA SILVA; PAMPHILE, JOÃO ALENCAR. Fungos de interesse: aplicações biotecnológicas. Revista UNINGÁ Review, v. 21, n. 1, 2015.

DEY, PINAKI et al. Improved production of cellulase by Trichoderma reesei (MTCC 164) from coconut mesocarp-based lignocellulosic wastes under response surface-optimized condition. 3 Biotech, v. 8, n. 9, p. 1-13, 2018.

EL-BAKRY, MAMDOUH et al. From wastes to high value added products: novel aspects of SSF in the production of enzymes. Critical Reviews in Environmental Science and Technology, v. 45, n. 18, p. 1999-2042, 2015.

FALKOSKI, DANIEL LUCIANO et al. Chrysoporthe cubensis: a new source of cellulases and hemicellulases to application in biomass saccharification processes. Bioresource technology, v. 130, p. 296-305, 2013.

FLORENCIO, CAMILA et al. Desafios relacionados à produção e aplicação das enzimas celulolíticas na hidrólise da biomassa lignocelulósica. Química Nova, v. 40, n. 9, 2017.

GOMES, ARTHUR FILIPE SOUSA et al. Substract and temperature effect on xylanase production by Aspergillus fumigatus using low cost agricultural wastes. Bioscience Journal, v. 32, n. 4, 2016.

GOUKANAPALLE, PRAVEEN KUMAR REDDY et al. Optimization of cellulase production by a novel endophytic fungus Pestalotiopsis microspora TKBRR isolated from Thalakona forest. Cellulose, v. 27, p. 6299-6316, 2020. 
HU, YUNZI et al. Optimisation of fungal cellulase production from textile waste using experimental design. Process Safety and Environmental Protection, v. 118, p. 133-142, 2018.

IRFAN, M. et al. Utilization of peanut shells as substrate for cellulase production in submerged fermentation through Box-Behnken Design. International Journal of Biology and Chemistry, v. 12, n. 2, p. 28-39, 2020.

KASANA, RAMESH C.; GULATI, ARVIND. Cellulases from psychrophilic microorganisms: a review. Journal of basic microbiology, v. 51, n. 6, p. 572-579, 2011.

KUHAD, RAMESH CHANDER et al. Revisiting cellulase production and redefining current strategies based on major challenges. Renewable and Sustainable Energy Reviews, v. 55, p. 249-272, 2016.

KUMAR, ANIL et al. Production of cellulolytic enzymes by Myceliophthora thermophila and their applicability in saccharification of rice straw. Biomass Conversion and Biorefinery, $p$. $1-14,2020$.

KUMAR, BIKASH et al. Production, purification and characterization of an acid/alkali and thermo tolerant cellulase from Schizophyllum commune NAIMCC-F-03379 and its application in hydrolysis of lignocellulosic wastes. AMB Express, v. 8, n. 1, p. 1-16, 2018.

LI, YONGHAO et al. Overproduction of cellulase by Trichoderma reesei RUT C30 through batch-feeding of synthesized low-cost sugar mixture. Bioresource technology, v. 216, p. 503$510,2016$.

LIMAYEM, ALYA, and STEVEN C. RICKE. "Lignocellulosic biomass for bioethanol production: current perspectives, potential issues and future prospects." Progress in Energy and Combustion Science 38.4 (2012): 449-467.

LIU, JIAWEN et al. Comparative characterization of extracellular enzymes secreted by Phanerochaete chrysosporium during solid-state and submerged fermentation. International journal of biological macromolecules, v. 152, p. 288-294, 2020.

MARTINS, LEONARDO FARIA et al. Comparison of Penicillium echinulatum and Trichoderma reesei cellulases in relation to their activity against various cellulosic substrates. Bioresource technology, v. 99, n. 5, p. 1417-1424, 2008.

MONDAL, Subhadeep et al. Contemporaneous synthesis of multiple carbohydrate debranching enzymes from newly isolated Aspergillus fumigatus SKF-2 under solid state fermentation: A unique enzyme mixture for proficient saccharification of plant bioresources. Industrial Crops and Products, v. 150, p. 112409, 2020.

MONTEIRO, VALDIRENE NEVES; DO NASCIMENTO SILVA, ROBERTO. Aplicações industriais da biotecnologia enzimática. Revista processos químicos, v. 3, n. 5, p. 9-23, 2009.

NAIR, ANU SADASIVAN et al. Waste office paper: a potential feedstock for cellulase production by a novel strain Bacillus velezensis ASN1. Waste management, v. 79, p. 491-500, 2018. 
NAZIR, SOBIA et al. Utilization of Bombyx ceiba Seed Pods: A novel substrate for cellulase production through solid state fermentation using response surface methodology. Punjab University Journal of Zoology, v. 34, n. 2, p. 213-219, 2019.

RAMAMOORTHY, NAVNIT KUMAR; SAMBAVI, T. R.; RENGANATHAN, SAHADEVAN. A study on cellulase production from a mixture of lignocellulosic wastes. Process Biochemistry, v. 83, p. 148-158, 2019.

RANA, VANDANA et al. On-site enzymes produced from Trichoderma reesei RUT-C30 and Aspergillus saccharolyticus for hydrolysis of wet exploded corn stover and loblolly pine. Bioresource technology, v. 154, p. 282-289, 2014.

RAWAT, REKHA et al. Generating fermentable sugars from rice straw using functionally active cellulolytic enzymes from Aspergillus niger HO. Energy \& Fuels, v. 28, n. 8, p. 50675075, 2014.

ROVEDA, MIRELA; HEMKEMEIER, MARCELO; COLLA, LUCIANE MARIA. Avaliação da produção de lipases por diferentes cepas de microrganismos isolados em efluentes de laticínios por fermentação submersa. Food Science and Technology, v. 30, n. 1, p. 126-131, 2010.

SADH, PARDEEP KUMAR; DUHAN, SUREKHA; DUHAN, JOGINDER SINGH. Agroindustrial wastes and their utilization using solid state fermentation: a review. Bioresources and Bioprocessing, v. 5, n. 1, p. 1-15, 2018.

SALAZAR, LUDMILA NOSKOSKI et al. Production, Partial Characterization and Application of Cellulases by Newly Isolated Penicillium sp. Using Agro-Industrial Substrate Solid-State Fermentation. Industrial Biotechnology, v. 15, n. 2, p. 79-88, 2019.

SANTOS, PRISCILA SOUZA DOS et al. Fermentação em estado sólido em resíduos agroindustriais para a produção de enzimas: uma revisão sistemática. The Journal of Engineering and Exact Sciences - JCEC, V. 4, N. 2, p. 1-12, 2018.

SATEESH, LANKA et al. Simultaneous cellulase production, saccharification and detoxification using dilute acid hydrolysate of S. spontaneum with Trichoderma reesei NCIM 992 and Aspergillus niger. Indian Journal of Microbiology, v. 52, n. 2, p. 258-262, 2012.

SHARMA, REETIKA et al. Improved Production of Multi-component Cellulolytic Enzymes Using Sweet Sorghum Bagasse and Thermophilic Aspergillus terreus RWY Through Statistical Process Optimization. Waste and Biomass Valorization, p. 1-15, 2019.

SHAWKY, BAHAA T. et al. Optimization of Cellulolytic enzymes production from local potent fungi using full factorial design for bioethanol production from MFEX-treated rice straw using Klebsiella oxytoca P2. BIOSCIENCE RESEARCH, v. 15, n. 1, p. 250-261, 2018.

SHELDON, ROGER A.; VAN PELT, SANDER. Enzyme immobilisation in biocatalysis: why, what and how. Chemical Society Reviews, v. 42, n. 15, p. 6223-6235, 2013.

SILVA, A. F. V. et al. Cellulase production to obtain biogas from passion fruit (Passiflora edulis) peel waste hydrolysate. Journal of Environmental Chemical Engineering, v. 7, n. 6, p. 103510, 2019. 
SINGH, ANITA et al. Optimization of cellulolytic enzyme production by thermophilic fungus Thermoascus aurantiacus using response surface methodology. Indian Journal of Biochemistry and Biophysics (IJBB), v. 56, n. 5, p. 399-403, 2019.

SINGHANIA, REETA RANI et al. Advancement and comparative profiles in the production technologies using solid-state and submerged fermentation for microbial cellulases. Enzyme and Microbial Technology, v. 46, n. 7, p. 541-549, 2010.

SIROHI, RANJNA et al. Cellulase production from pre-treated pea hulls using Trichoderma reesei under submerged fermentation. Waste and Biomass Valorization, v. 10, n. 9, p. 2651$2659,2019$.

SOARES, IZABEL APARECIDA et al. Identificação do potencial amilolítico de linhagens mutantes do fungo filamentoso Aspergillus nidulans. Food Science and Technology, v. 30, n. 3, p. 700-705, 2010.

SOCCOL, CARLOS RICARDO et al. Recent developments and innovations in solid state fermentation. Biotechnology Research and Innovation, v. 1, n. 1, p. 52-71, 2017.

SUBSAMRAN, KHAJEEWAN et al. Potential use of vetiver grass for cellulolytic enzyme production and bioethanol production. Biocatalysis and Agricultural Biotechnology, v. 17, p. 261-268, 2019.

SUN, WEI-CHENG; CHENG, CHUNG-HSIEN; LEE, WEN-CHIEN. Protein expression and enzymatic activity of cellulases produced by Trichoderma reesei Rut C-30 on rice straw. Process Biochemistry, v. 43, n. 10, p. 1083-1087, 2008.

SUTO, MANABU; TOMITA, FUSAO. Induction and catabolite repression mechanisms of cellulase in fungi. Journal of bioscience and bioengineering, v. 92, n. 4, p. 305-311, 2001.

TAI, WAN YI et al. Comprehensive studies on optimization of cellulase and xylanase production by a local indigenous fungus strain via solid state fermentation using oil palm frond as substrate. Biotechnology progress, v. 35, n. 3, p. e2781, 2019.

THOMAS, LEYA; LARROCHE, CHRISTIAN; PANDEY, ASHOK. Current developments in solid-state fermentation. Biochemical Engineering Journal, v. 81, p. 146-161, 2013.

VERMA, NITIN; KUMAR, VIVEK. Application of Box-Behnken design for the optimization of cellulase production under solid-state fermentation. SN Applied Sciences, v. 1, n. 12, p. 1$10,2019$.

VERMA, NITIN; KUMAR, VIVEK. Impact of process parameters and plant polysaccharide hydrolysates in cellulase production by Trichoderma reesei and Neurospora crassa under wheat bran based solid state fermentation. Biotechnology Reports, v. 25, p. e00416, 2020.

YOON, LI WAN et al. Fungal solid-state fermentation and various methods of enhancement in cellulase production. Biomass and bioenergy, v. 67, p. 319-338, 2014.

ZHOU, WENTING et al. Feasibility of lipid production from waste paper by the oleaginous yeast Cryptococcus curvatus. BioResources, v. 12, n. 3, p. 5249-5263, 2017. 
ZNAMEROSKI, ELIZABETH A. et al. Induction of lignocellulose-degrading enzymes in

Neurospora crassa by cellodextrins. Proceedings of the National Academy of Sciences, v. 109, n. 16, p. 6012-6017, 2012. 